\title{
Zur inklusiven Betreuung von Kindern mit (drohender) Behinderung in Kindertageseinrichtungen in Bayern und im Rheinland
}

\author{
Ein Studienvergleich \\ Rüdiger Kißgen', Claudia Wirts², Daniela Limburg', \\ Monika Wertfein ${ }^{2}$, Sebastian Franke', Janina Wölfl' ${ }^{2}$, Julia Austermühle ${ }^{1}$ \\ 1 Universität Siegen \\ 2 Staatsinstitut für Frühpädagogik München
}

\begin{abstract}
Zusammenfassung: Es werden zwei Studien vorgestellt, die die Rahmenbedingungen bei der Umsetzung von Inklusion im Elementarbereich untersuchen. Die Inklusion-vor-Ort-Studie beschäftigte sich mit dem Thema Inklusion als gemeinsame Aufgabe von Kindertageseinrichtungen und Frühförderung in Bayern. Die Rheinland-Kita-Studie explorierte die inklusive Betreuungssituation in den Kindertageseinrichtungen des Rheinlandes. Die Ergebnisse zeigen einen deutlichen Anteil an Kindertageseinrichtungen, die keine Kinder mit (drohender) Behinderung betreuen. Mit Blick auf alle - an den beiden Studien teilnehmenden - Einrichtungen finden sich Hinweise auf Nachbesserungsbedarfe bezogen auf Aspekte wie Barrierefreiheit, Professionalisierung der Kita-Teams und Aufbau von tragfähigen Netzwerken.
\end{abstract}

Schlüsselwörter: Kinder mit Behinderung, Kinder mit drohender Behinderung, Risikokinder, Inklusion, Kindertageseinrichtung, Kooperation

\begin{abstract}
Inclusion of children with (impending) disabilities in daycare centers in Bavaria and the Rhineland - a comparison of two studies Summary: Two studies are presented that examine the conditions for the implementation of inclusion in kindergartens. The Inklusion-vor-Ort-Studie (on-site inclusion study) focused on inclusion as a common task of kindergartens and early intervention in Bavaria. The RheinlandKita-Studie (Rhineland Kita study) explored the inclusive childcare situation in kindergartens in the Rhineland. The results reveal a high proportion of day care centers for children that do not yet take care of children with (impending) disabilities. Overall, there seems to be a need for improvement regarding accessibility, professionalization of the daycare teams and sustainable networks for daycare facilities for children.
\end{abstract}

Keywords: Children with disabilities, children at risk of disabilities, children at risk, inclusion, kindergarten, cooperation

\section{Einleitung}

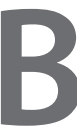

und und Länder stehen spätestens seit der Ratifizierung der UN-Behindertenrechtskonvention 2009 in der Verantwortung, die erfolgreiche Umsetzung von Inklusion in den verschiedenen Bildungssystemen, so auch in den Kindertageseinrichtungen (Kitas), zu unterstützen. Eine einheitliche Betrachtung der Thematik ist grundsätzlich schwierig, da alle 16 Bundesländer eigenen länderspezifischen Strukturen und gesetzlichen Rahmenbedingungen unterliegen (Brunner 2018). Deutschlandweit müssen Grundvoraussetzungen geschaffen werden, um die individuellen Bedürfnisse jedes Kindes berücksichtigen zu können (Auto- 
rengruppe Bildungsberichterstattung 2014). Die Inklusion-vor-Ort-Studie (IVO-Studie) für das Bundesland Bayern sowie die Rheinland-KitaStudie (RKS) für das Zuständigkeitsgebiet des Landschaftsverbandes Rheinland (LVR) ${ }^{1}$ im Bundesland Nordrhein-Westfalen (NRW) haben im Rahmen zweier Online-Befragungen den aktuellen Umsetzungsstand von Inklusion in Kitas untersucht. Für das Kita-Jahr 2015/2016 wurden in Bayern alle 8.748 Kitas (ISB 2015) über die Jugendämter kontaktiert. Im Rheinland wurden alle 5.220 Kitas, die für das Kita-Jahr 2017/ 2018 in KiBiz.web ${ }^{2}$ erfasst waren, zur Teilnahme eingeladen.

Die IVO-Studie in Bayern (Wirts et al. 2018, Wölfl et al. 2017) wurde durch das Staatsinstitut für Frühpädagogik in enger Kooperation mit dem Bayerischen Sozialministerium sowie der Arbeitsstelle Frühförderung Bayern konzipiert und durchgeführt. Die RKS (Kißgen 2019a/b, Kißgen et al. 2019) erfolgte im Auftrag des LVR. Zentrale Zielsetzungen beider Studien waren:

- Strukturen und Konzepte von Kooperation im inklusiven Kontext zu betrachten,

- Herausforderungen und Möglichkeiten für den bedarfsgerechten Ausbau von inklusiven Betreuungsplätzen für Kinder mit (drohender) Behinderung herauszuarbeiten und

- Gelingensbedingungen für eine inklusive Bildung, Erziehung und Betreuung im Elementarbereich zu untersuchen.

Inklusive Bildung - hier verstanden als gleichberechtigte, gemeinsame Teilhabe von Kindern mit und ohne Behinderung - wird in deutschen Kitas vielerorts bereits in vielfältiger Weise gelebt und umgesetzt. Vor allem jedoch in KitaTeams, die noch über keine Erfahrungen mit der gemeinsamen Bildung, Erziehung und Betreuung von Kindern mit und ohne Behinderung verfügen, gibt es häufig noch emotionale sowie organisatorische Hürden bei der Aufnahme von Kindern mit Behinderung (KmB). Inwiefern Inklusion in den Einrichtungen vor Ort für alle Kinder und Familien tatsächlich gelingen kann, hängt sehr davon ab, ob den Kitas ein tragfähiges Netzwerk aus Unterstützungsoptionen zur Verfügung steht und wie gut die Zusammenarbeit und der Abruf von Unterstützungsangeboten funktioniert (Wirts et al. 2015, Wertfein und Wirts 2016).

Der vorliegende Beitrag strebt eine vergleichende Betrachtung der quantitativen Ergebnisse der Studien aus Bayern und dem Rheinland an. Hierzu wurden jene Aspekte ausgewählt, die aufgrund einer vergleichbaren Abfrage eine Gegenüberstellung ermöglichen. Dazu zählen neben dem Blick auf die betreuten Kinder und die Ausstattungsmerkmale in den Kitas auch die Betrachtung ihrer Vernetzung und Kooperationsmöglichkeiten. Der Ergebnisvergleich beider Studien im Hinblick auf Gemeinsamkeiten und Unterschiede soll dazu dienen, bewährte Strukturen und Gelingensfaktoren sowie noch bestehende Hürden zu verdeutlichen, die bundeslandübergreifende Relevanz haben.

\section{Studien-Designs}

\section{IVO-Studie Bayern}

Die IVO-Studie umfasste neben der hier vorgestellten Befragung von Kita-Leitungen auch Befragungen von Frühförderstellen-Leitungen und Mitarbeitenden der (staatlich geförderten) mobilen Heilpädagogischen Fachdienste (mHFD) (Ergebnisse hierzu vgl. Wirts et al. 2018). Die Befragung der Kita-Leitungen wurde mithilfe eines Online-Fragebogens durchgeführt und beinhaltete folgende Themenbereiche:

- Angaben zur Person, zur Einrichtung, zum Kita-Team und zu Kindern mit besonderem Unterstützungsbedarf,

- Organisatorische Umsetzung und Finanzierung von Leistungen,

- Kooperation und Netzwerkarbeit von Kitas im Überblick, 
- Zusammenarbeit mit ausgewählten Kooperationspartnerinnen und -partnern: Interdisziplinäre Frühförderstellen (IFF) und mobile Heilpädagogische Fachdienste (mHFD) und

- die inklusive Haltung des Kita-Teams.

Im Fragebogen kamen größtenteils geschlossene Antwortformate zur Anwendung. Diese wurden an einigen Stellen um offene Fragen ergänzt. Die bayernweit angelegte Befragung von Kita-Leitungen erfolgte von Mitte Mai bis Mitte Juni 2016.

\section{Rheinland-Kita-Studie}

Die Rheinland-Kita-Studie setzte sich aus einer quantitativ-empirischen Hauptuntersuchung und einer sich anschließenden qualitativ-empirischen Vertiefungsstudie zusammen. Betrachtet werden in diesem Beitrag die Ergebnisse der Hauptuntersuchung. Die Online-Befragung der Kita-Leitungen im Rheinland fand im Oktober 2017 statt und beinhaltete Fragen zu vier Themenbereichen:

- Angaben zu den Kindern, die die Kitas besuchten, sowie zum Umfang und zur Verwendung der finanziellen Mittel, die die Einrichtung im Zusammenhang mit der Betreuung von $\mathrm{KmB}$ erhält,

- Angaben zu Eigenschaften und Ausstattung der Einrichtungen,

- Betrachtung der Zusammensetzung und vorhandenen Qualifizierungen der Kita-Teams und

- Angaben zu den Kita-Leitungen.

Ähnlich der IVO-Studie bestand der Fragebogen hauptsächlich aus geschlossenen Items. Durch offene Frageformate am Ende eines jeweiligen Themenfeldes hatten die Kita-Leitungen zusätzlich die Gelegenheit, eigene Anmerkungen und Gedanken zu äußern. Die wichtigsten Ergebnisse wurden in einem Abschlussbericht zur RKS sowie in Fachartikeln veröffentlicht (Austermühle et al. 2019, Kißgen et al. 2019, Kißgen et al. im Druck).

\section{Theoretischer Hintergrund und gemeinsame Fragestellungen}

Für die vergleichende Ergebnisbetrachtung der beiden Studien werden vier Themenfelder in den Fokus genommen:

Das erste Themenfeld richtet den Blick auf die Kinder in den inklusiven Kitas ${ }^{3}$. Dabei werden verschiedene Behinderungsarten unterschieden. Ein weiterer Fokus wird auf Kinder gelegt, die nach Mayr und Held (2010) als Risikokinder gelten. Mit diesem Begriff sind Kinder charakterisiert, die deutlich hinsichtlich ihrer Entwicklung, ihres Verhaltens, ihres Gesundheitszustandes oder ihrer familiären und sozialen Situation auffällig sind, ohne dass dies als eine (drohende) Behinderung im sozialrechtlichen Sinn anzusehen ist. Bislang wird in der Forschungsliteratur wenig betrachtet, welchen besonderen Betreuungsbedarf diese Kinder mit sich bringen und welche Herausforderungen hieraus für die pädagogischen Fachkräfte entstehen.

Das zweite Themenfeld beleuchtet die aktuellen Zugangsmöglichkeiten für KmB im Sinne von Barrierefreiheit in den Kitas aus Sicht der Kita-Leitungen. Die Barrierefreiheit von Einrichtungen wird als Qualitätsindikator inklusiver Kitas aufgefasst (Behr 2009, Brunner 2018, Heimlich 2013).

Das dritte Themenfeld nimmt weitere Qualitätsindikatoren in den Blick. Diese betreffen die strukturellen Voraussetzungen inklusiver Kitas (Behr 2009, Brunner 2018, Heimlich 2013). Dabei richtet sich der Blick auch auf die Kita-Leitungen mit der Frage, ob diese vom Gruppendienst für Leitungs- und konzeptuelle Aufgaben im Zusammenhang mit Inklusion freigestellt sind. Schelle (2017) stellt die Rolle der Kita-Leitung als Schlüsselposition bei der Umsetzung von inklusiven Konzepten heraus. Damit verbunden sind komplexe Anforderungen, die zusätz- 
lich zum pädagogischen Gruppendienst und der Leitungsfunktion konzeptuelle und organisationsbezogene Aufgaben beinhalten (Nentwig-Gesemann et al. 2016). Weiterhin wird die Zusammensetzung der Professionen innerhalb der Kita-Teams unter Berücksichtigung der fachlichen Qualifizierung im Hinblick auf Inklusion in den Kitas in diesem Themenfeld betrachtet. Die Umsetzung inklusiver Bildung erfordert eine entsprechende Expertise (Brunner 2018). Schließlich wird die von den Kita-Leitungen wahrgenommene Haltung zu Inklusion innerhalb der Kita-Teams dargestellt. Diese zeigt, wie aufgeschlossen sie ihre pädagogischen Fachkräfte gegenüber Inklusion einschätzen. Damit einhergehend wird betrachtet, auf welche Weise die Beschäftigung mit dem Thema Inklusion in den Kitas erfolgt.

Das vierte Themenfeld beschäftigt sich mit der Frage, welche Kooperationsoptionen den Kitas unterstützend bei der Umsetzung von Inklusion zur Verfügung stehen und wie zufrieden die Kitas mit den verfügbaren Kooperationsoptionen sind. Zur Vielzahl denkbarer Kooperationsoptionen zählen Frühförderstellen, therapeutische Fachkräfte mit unterschiedlichen Professionen oder Heilpädagoginnen und Heilpädagogen. Im Rheinland gewinnen Fachberatungen immer mehr an Bedeutung und etablieren sich zunehmend im Beratungs- und Unterstützungssystem für Kitas (Hruska 2018). Fachberatungen können sowohl bei Jugendämtern als auch bei Trägern verortet sein. Obwohl es für Fachberatungen noch kein einheitliches Tätigkeitsprofil gibt und ihre Aufgaben recht unterschiedlich sein können, sind sie auch für die Hilfestellung bei der Umsetzung von Inklusion zuständig, bei der einer multiprofessionellen Ausrichtung in der pädagogischen Arbeit eine hohe Bedeutung zukommt (Brunner 2018, Heimlich 2013). Gerade wenn Multiprofessionalität innerhalb der Kita-Teams wenig bis gar nicht vorhanden ist, können externe Systeme eine kompensierende Funktion übernehmen.

\section{Stichprobenbeschreibungen}

\section{Rücklaufquoten}

Insgesamt wurden in der bayerischen IVO-Studie die Antworten von 2.823 Kita-Leitungen in die Analysen miteinbezogen. Bei 8.748 Kitas in ganz Bayern (ISB 2015) entspricht das einer Rücklaufquote von rund $32,3 \%$. Fragebögen von heilpädagogischen Sondereinrichtungen wie z. B. schulvorbereitende Einrichtungen (SVE) und von Hort-Einrichtungen wurden für die hier präsentierten Analysen ausgeschlossen. Einbezogen in die Auswertungen wurden alle Fragebögen, die mindestens bis zur Cut-offFrage $^{4}$ zur Betreuung von KmB beantwortet wurden. Vergleichsdaten aus dem Bildungsbericht Bayern (ISB 2015) und des Statistischen Landesamtes Bayern (2015) zeigen, dass die Stichprobe hinsichtlich Trägerverteilung, Einrichtungsarten, Altersstruktur und pädagogischer Ansätze weitgehend den Verteilungen in Bayern entspricht. Es ergibt sich lediglich eine leichte Überrepräsentation von Kitas aus kleineren Gemeinden (Wölfl et al. 2017).

In der RKS wurde ein Fragebogen als bearbeitet gewertet, wenn mindestens $50 \%$ der Fragen beantwortet worden waren. Dies war bei 1.681 Fragebögen der Fall. Da 5.034 Einrichtungen angeschrieben wurden, lag die Rücklaufquote somit bei 33,4\%. Die zur Verfügung gestellten Vergleichsdaten aus KiBiz.web zeigen, dass die Stichprobe bezüglich ihrer Lage ${ }^{5}$ gut mit der Verteilung im Rheinland übereinstimmt.

\section{Einrichtungscharakteristika}

In beiden Studien waren die inklusiven Einrichtungen deutlich überrepräsentiert. In Bayern lag der prozentuale Anteil der Einrichtungen, die mindestens ein KmB betreuen, 2014 bei $25 \%$ (ISB 2015), in der IVO-Studie waren 
es $50 \%$ der teilnehmenden Einrichtungen. $58 \%$ der Einrichtungen, die an der RKS teilnahmen, betreuten, bereits mindestens ein KmB. Laut Jugendhilfestatistik lag der Anteil an Einrichtungen, die mindestens ein Kind mit Eingliederungshilfe nach SGB VIII oder XII betreuen, für die Jugendamtsbezirke der LVR Mitgliedskörperschaften im Jahr 2017 bei 34,2\% (IT NRW Ref.512.Soziales.KJH) ${ }^{6}$. Die Überrepräsentation inklusiver Einrichtungen im Vergleich mit Einrichtungen ohne $\mathrm{KmB}$ in beiden Studien kann vermutlich durch deren selektiv größeres Interesse an der Befragung erklärt werden.

\section{Kita-Leitungen}

In der IVO-Studie ist der Großteil der teilnehmenden Kita-Leitungen (97\%) weiblich. Die meisten Kita-Leitungen (60\%) gaben an, über ein (Fach-)Abitur oder einen vergleichbaren Abschluss zu verfügen, $39 \%$ weisen einen mittleren Bildungsabschluss (z. B. Mittlere Reife) auf und $1 \%$ einen (qualifizierten) Hauptschulabschluss. Bei den Fragen zur beruflichen Qualifikation und zu Studienabschlüssen waren Mehrfachnennungen möglich. Der mit Abstand größte Teil der befragten Kita-Leitungen (90\%) hat einen Abschluss als Erzieherin oder Erzieher, $12 \%$ der Befragten haben einen Hochschulabschluss.

In der RKS sind 95\% der Kita-Leitungen von Einrichtungen mit oder ohne KmB weiblich. Von den Leitungen inklusiver Kitas verfügen $51 \%$ über das (Fach-)Abitur und $49 \%$ über die Mittlere Reife oder einen vergleichbaren Abschluss. Etwas weniger als die Hälfte der Leitungen von Kitas ohne KmB (47\%) haben die Schullaufbahn mit dem (Fach-)Abitur, $53 \%$ mit der Mittleren Reife oder einem vergleichbaren $A b$ schluss beendet. $88 \%$ aller teilnehmenden Leitungen haben eine Ausbildung als Erzieherin oder Erzieher. Rund $17 \%$ verfügen über einen Hochschulabschluss.

\section{Vergleichende Ergebnisdarstellung}

Die folgende deskriptive Darstellung der Ergebnisse stellt die vergleichbaren Daten der Fragebogenerhebungen zu den zuvor skizzierten vier Themenfeldern vor. Jeweils an deren Ende erfolgt eine kurze Ergebnisdiskussion.

\section{Kinder in den inklusiven Kitas}

\section{Kinder mit diagnostizierter (drohender) Behinderung}

Tabelle 1 enthält eine Übersicht zu den Behinderungsarten der in den inklusiv arbeitenden Kitas betreuten $\mathrm{KmB}$. In beiden Studien betreuen die meisten Kitas Kinder mit Verhaltensauffälligkeiten und Allgemeiner Entwicklungsverzögerung, während deutlich weniger Kitas auch Kinder mit Mehrfachbehinderung, Sinnesbehinderungen, chronischen Krankheiten und geistiger Behinderung betreuen. Mehrfachangaben waren in beiden Studien möglich.

\section{Risikokinder}

In beiden Studien gaben rund $71 \%$ der Kita-Leitungen an, mindestens ein sogenanntes Risikokind in ihrer Einrichtung zu betreuen. In der IVO-Studie waren dies $82,5 \%$ der inklusiven Einrichtungen gegenüber 58,7\% der Einrichtungen ohne KmB. Dieser Unterschied wird signifikant $\left(\mathrm{X}_{\mathrm{df}=1}^{2}=174.32 ; \mathrm{p}<.001 ; \varphi=0.26\right)$. In der RKS gaben $84 \%$ der Leitungen inklusiver Einrichtungen an, auch Risikokinder zu betreuen, während es in den Einrichtungen ohne $\mathrm{KmB}$ lediglich $54 \%$ waren $\left(X_{d f=1}^{2}=185.71 ; p<.001\right.$; $\varphi=0.33$ ). Die in beiden Studien beschriebenen Auffälligkeiten der Risikokinder bezogen sich häufig auf deren Sprachentwicklung, Sozialverhalten und Emotionsregulation. Ebenfalls häufig genannt wurden Auffälligkeiten und Störungen in den Bereichen der Wahrnehmung, Motorik sowie kognitive oder allgemeine Entwicklungsverzögerungen. 
Tab. 1: Behinderungsarten der in inklusiv arbeitenden Kitas betreuten Kinder mit (drohender) Behinderung

\begin{tabular}{|l|c|c|c|c|}
\hline \multirow{2}{*}{ Behinderungsart } & \multicolumn{2}{|c|}{ IVO } & \multicolumn{2}{|c|}{ RKS } \\
\cline { 2 - 5 } & $\mathbf{N}_{\text {Kitas }}$ & \% aller IVO-Kitas & N $_{\text {Kitas }}$ & \% aller RKS-Kitas \\
\hline Verhaltensstörung & 689 & 24 & 320 & 19 \\
Allg. Entwicklungsverzögerung & 684 & 24 & 454 & 27 \\
Mehrfachbehinderung & 335 & 12 & 212 & 13 \\
Sprachbehinderung & 252 & 9 & 172 & 10 \\
Körperbehinderung & 209 & 7 & 149 & 9 \\
Chronische Krankheit & 199 & 7 & 102 & 10 \\
Geistige Behinderung & 196 & 7 & 173 & 4 \\
Hörbehinderung & 153 & 5 & 68 & 3 \\
Sehbehinderung & 104 & 4 & 50 & \\
\hline
\end{tabular}

Anmerkung: Anzahl antwortender inklusiver Kitas der IVO-Studie: N=788; Anzahl antwortender inklusiver Kitas der RKS: $\mathrm{N}_{\min }=756-\mathrm{N}_{\max }=846$

\section{Diskussion zu Themenfeld 1}

Es lässt sich feststellen, dass inklusiv arbeitende Kitas am häufigsten Kinder mit Verhaltensstörungen oder allgemeinen Entwicklungsverzögerungen betreuen. Alle anderen Behinderungsarten sind deutlich seltener vertreten. Erklärend könnte hierfür sein, dass Kinder mit Verhaltensstörungen oder allgemeinen Entwicklungsverzögerungen häufig erst nach erfolgter Aufnahme in eine Regel-Kita erkannt und diagnostiziert werden.

Auffällig sind die Angaben zu den Risikokindern in beiden Studien. Bei diesen Kindern liegt keine diagnostizierte Behinderung vor. Jedoch zeigt sich ausgehend von den Beschreibungen der Auffälligkeiten ein besonderer Betreuungsbedarf, der im Rahmen von inklusiven Einrichtungskonzepten mitzudenken ist. Aufgrund des fehlenden sozialrechtlichen Status erhalten die Kitas bei der Betreuung von Risikokindern keine zusätzlichen finanziellen Mittel bzw. können keine Gruppenstärkereduzierungen vornehmen. In beiden Studien fällt auf, dass von Einrichtungen mit KmB signifikant häufiger angegeben wird, Risikokinder zu betreuen, als von Einrichtungen ohne KmB. Gründe für diesen Unterschied können aus den vorliegenden Daten leider nicht erschlos- sen werden, allerdings lassen sich die beiden folgenden Effekte vermuten: Zum einen eine höhere Sensibilisierung in den inklusiven Einrichtungen bezüglich der Förderbedarfe von Kindern und zum anderen die zugesprochene Kompetenz im Umgang mit förderbedürftigen Kindern, sodass diese häufiger in inklusiven Einrichtungen angemeldet werden. Einschränkend muss beachtet werden, dass die Kinder ohne Diagnose seitens der Einrichtungsleitungen als "Risikokinder" eingestuft wurden und daher subjektive Kategorien zur Einordnung herangezogen wurden, die stark variieren können.

\section{Barrierefreiheit}

Unabhängig davon, ob sie bereits $\mathrm{KmB}$ betreuen oder nicht, wurden die Kita-Leitungen bezogen auf die Barrierefreiheit in ihren Einrichtungen gebeten, eine Einschätzung vorzunehmen. Wie Tabelle 2 zu entnehmen ist, ist die Barrierefreiheit je nach Behinderungsart sehr unterschiedlich (22\% bis $52 \%$ ). Für Kinder mit Sehbehinderungen werden am häufigsten Barrieren angegeben, gefolgt von Kindern mit Körperbehinderungen, seltener für Kinder mit Hörbehinderung oder geistiger Behinderung. 
Tab. 2: Barrierefreiheit für Kinder mit Behinderung differenziert nach Behinderungsart

\begin{tabular}{|l|l|c|c|c|c|}
\hline \multicolumn{2}{|l|}{ Behinderungsart } & N & ja (\%) & teilweise (\%) & nein (\%) \\
\hline \multirow{3}{*}{ Sehbehinderung } & IVO & 2.645 & 22 & 40 & 38 \\
\cline { 2 - 6 } & RKS & 1.548 & 27 & 42 & 31 \\
\hline \multirow{3}{*}{ Körperbehinderung } & IVO & 2.782 & 35 & 33 & 32 \\
\cline { 2 - 6 } & RKS & 1.588 & 40 & 32 & 28 \\
\hline \multirow{3}{*}{ Hörbehinderung } & IVO & 2.664 & 41 & 34 & 25 \\
\cline { 2 - 6 } & RKS & 1.544 & 52 & 34 & 15 \\
\hline \multirow{3}{*}{ Geistige Behinderung } & IVO & 2.689 & 45 & 35 & 15 \\
\cline { 2 - 6 } & RKS & 1.541 & 50 & & 33 \\
\hline
\end{tabular}

\section{Diskussion zu Themenfeld 2}

Die Beschäftigung mit dem Thema Barrierefreiheit ist offensichtlich immer noch ein zentraler Aspekt in der inklusiv werdenden KitaLandschaft. Worin die Barrieren im Einzelnen genau bestehen, wurde in beiden Studien nicht explizit erhoben. Denkbar wären bauliche Aspekte bezogen auf vorhandene Räumlichkeiten und räumliche Zugangsmöglichkeiten oder auch Aspekte bezogen auf die behindertengerechte räumliche Ausstattung bzw. geeignetes Material. Möglicherweise kann Barrierefreiheit bei bestimmten Behinderungsarten (vermeintlich) einfacher bzw. mit weniger Aufwand oder Ressourceneinsatz umgesetzt werden, dies müssen weitere Studien zeigen. Aus den Anmerkungen der Kita-Leitungen der RKS zu dieser Frage ergeben sich darüber hinaus Hinweise, dass das Wissen darüber fehlt, was Barrierefreiheit in Bezug auf bestimmte Behinderungsbilder überhaupt bedeutet (Kißgen et al. 2019). Zukünftige Studien sollten daher verstärkt auf eine praxisnahe Operationalisierung bei der Erfassung von Barrierefreiheit achten. Schulungen und weitere Aufklärung über die Bedeutung von Barrierefreiheit für spezifische Behinderungsbilder erscheinen somit dringend notwendig.

\section{Strukturelle Voraussetzungen inklusiver Kitas und Haltung zum Thema Inklusion}

\section{Freistellung der Kita-Leitungen vom Gruppendienst}

Im Vergleich der beiden Studien ergibt sich ein deutlicher Unterschied im Hinblick auf die Freistellung der Kita-Leitungen inklusiver Einrichtungen vom Gruppendienst. Während in der IVO-Studie lediglich $12 \%$ der Kita-Leitungen angeben, ganz freigestellt zu sein, trifft dies im Rheinland auf $41 \%$ der Leitungen zu. Auch die teilweise Freistellung kommt im Rheinland mit $45 \%$ deutlich häufiger vor als in Bayern (29\%). Eine genauere Analyse zeigt, dass Kita-Leitungen kleinerer Einrichtungen (bis 45 Kinder) in der IVO-Studie zu $79 \%$ nicht freigestellt sind, während dies bei großen Einrichtungen ( $>76$ Kinder) nur $40 \%$ betrifft. In der RKS sind $27 \%$ der Kita-Leitungen kleinerer und nur 7\% der großen Einrichtungen nicht freigestellt. Der Vergleich von Einrichtungen mit $\mathrm{KmB}$ und Einrichtungen ohne $\mathrm{KmB}$ ergibt sowohl für die IVOStudie $\left(X_{d f=2}^{2}=29.07 ; p<.001 ; \varphi=0.115\right)$ als auch für die RKS $\left(X_{d f=1}^{2}=25.80 ; p<.001 ; \varphi=0.13\right)$, dass Kita-Leitungen in Einrichtungen mit KmB hoch signifikant häufiger vom Gruppendienst freigestellt sind als in Einrichtungen ohne KmB. 


\section{Zusammensetzung der Kita-Teams}

In beiden Studien setzen sich die Kita-Teams größtenteils aus Erzieherinnen und Erziehern bzw. allgemein pädagogisch qualifiziertem Personal zusammen. Der Anteil von spezifisch ausgebildeten Fachkräften fällt eher gering aus.

In Bayern liegt der Anteil an allgemein pädagogisch qualifizierten Mitarbeiterinnen und Mitarbeitern in inklusiven Einrichtungen bei $83 \%$ und in Einrichtungen, die keine $\mathrm{KmB}$ betreuen, bei $86 \%$. Zu dieser Gruppe wurden in der IVOStudie Erzieherinnen und Erzieher, Kinderpflegerinnen und Kinderpfleger, Ergänzungskräfte sowie andere pädagogische Mitarbeiterinnen und Mitarbeiter zusammengefasst. Der Anteil des "spezifisch qualifizierten" Personals ${ }^{7}$ liegt in Einrichtungen, die $\mathrm{KmB}$ betreuen, bei $7 \%$ und in den nicht inklusiven Einrichtungen bei $2 \%$.

Im Rheinland stellen in beiden Einrichtungsarten Erzieherinnen und Erzieher die größte Gruppe innerhalb der Kita-Teams dar (Kitas mit $\mathrm{KmB}: 65 \%$; Kitas ohne KmB: $72 \%$ ). Mit $6 \%$ in inklusiven Einrichtungen bzw. $5 \%$ in Einrich- tungen, die noch nicht inklusiv arbeiten, haben Pädagoginnen und Pädagogen ${ }^{8}$ mit einem Hochschulabschluss einen eher geringen Anteil am Team. Hinsichtlich der Festanstellung von Fachtherapeutinnen und -therapeuten ergibt sich für inklusive Einrichtungen ein Anteil von lediglich $3 \%$. In Einrichtungen, die keine $\mathrm{KmB}$ betreuen, arbeiten keine Therapeutinnen und Therapeuten.

\section{Haltung der Kita-Teams zum Thema Inklusion}

In beiden Studien wurden die Kita-Leitungen gebeten, die Haltung innerhalb ihrer Teams bezogen auf das Thema Inklusion auf einer 5-Punkt-Skala von sehr zurückhaltend bis sehr aufgeschlossen einzuschätzen. Sowohl in Einrichtungen, die bereits $\mathrm{KmB}$ betreuen, als auch in Einrichtungen, die noch keine $\mathrm{KmB}$ aufgenommen haben, wird der größte Anteil der pädagogischen Teams eher aufgeschlossen gegenüber Inklusion eingeschätzt (vgl. Abb. 1 und 2). Es wird jedoch auch deutlich, dass die Haltung innerhalb von Kita-Teams in Einrich-

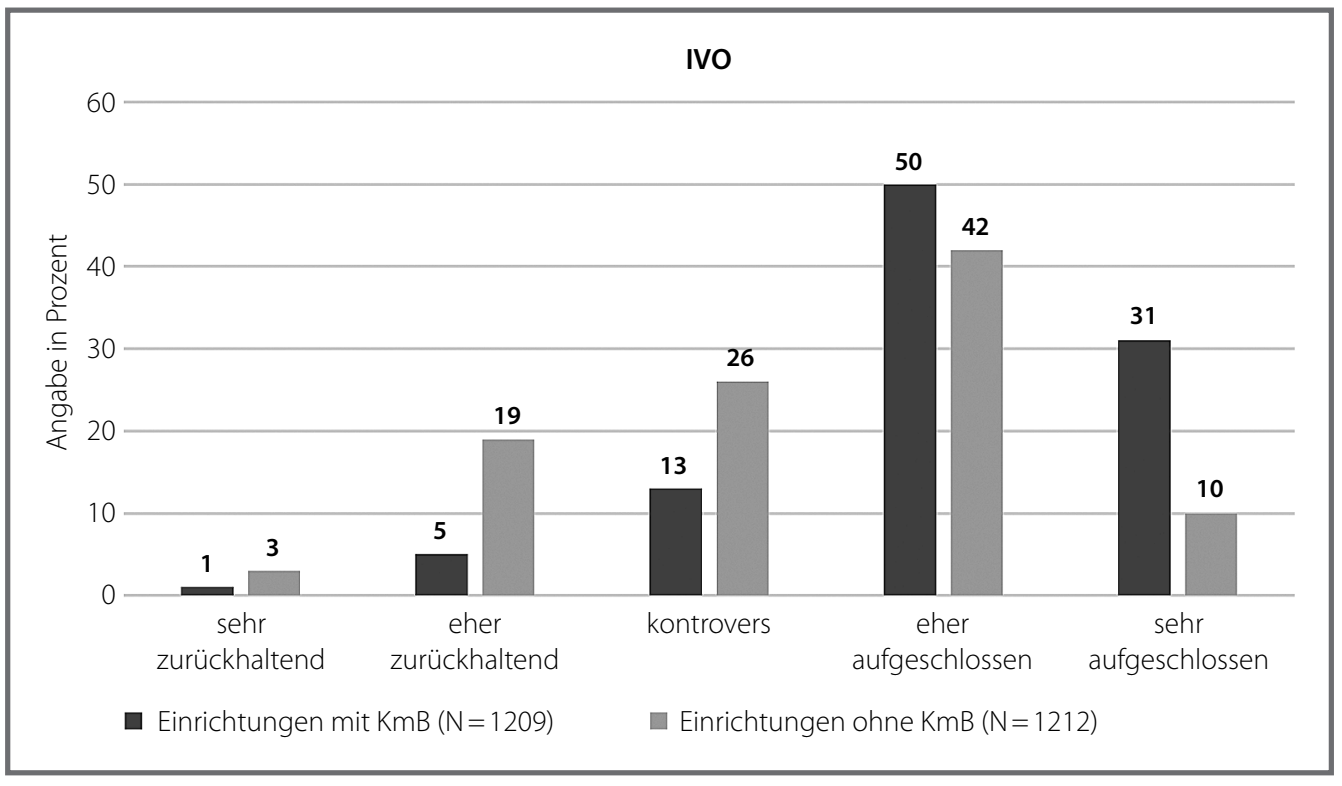

Abb. 1: Haltung der Kita-Teams zu Inklusion aus Sicht der Kita-Leitungen (IVO)

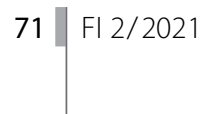




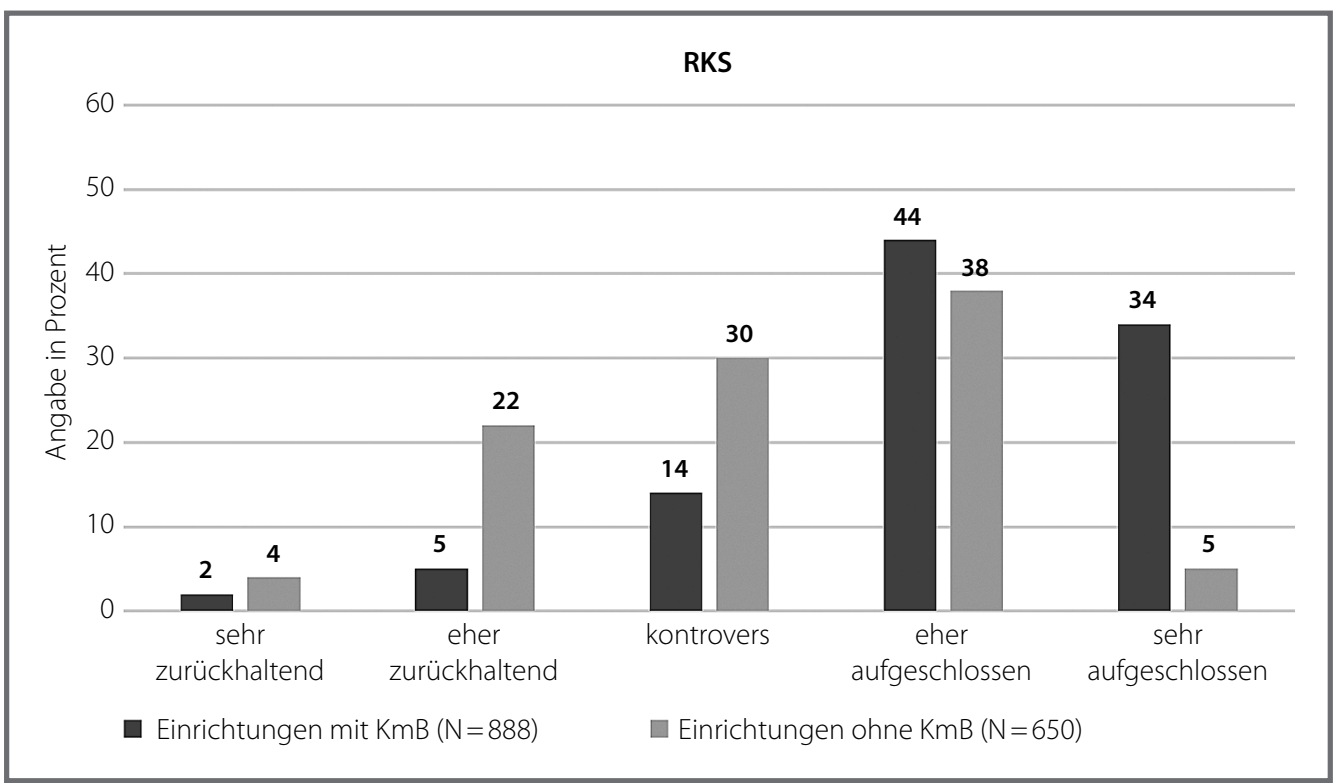

Abb. 2: Haltung der Kita-Teams zu Inklusion aus Sicht der Kita-Leitungen (RKS)

tungen, die noch keine KmB betreuen, im Vergleich weniger aufgeschlossen ist. Ein Mittelwertvergleich zeigt in beiden Studien, dass die eingeschätzte Aufgeschlossenheit des Teams für das Thema Inklusion in den Einrichtungen höher ist, die bereits mindestens ein $\mathrm{KmB}$ betreuen (IVO: $t=17,94 ; p<.001$; RKS: $t=17,16$; $\mathrm{p}<.001)$.

\section{Art der Beschäftigung mit dem Thema Inklusion}

Die Kita-Leitungen wurden in beiden Studien gefragt, auf welche Art und Weise die Auseinandersetzung mit dem Thema Inklusion erfolgt, sofern die Teams sich mit dem Thema schon auseinandergesetzt haben. In der IVOStudie gaben $40 \%$ der Leitungen von Einrichtungen ohne $\mathrm{KmB}$ an, sich im Team noch nicht mit dem Thema Inklusion beschäftigt zu haben. In der RKS-Stichprobe waren es $24 \%$. Insgesamt fällt auf, dass Kita-Leitungen der IVO-Studie höhere Werte in allen Kategorien erzielen (vgl. Abb. 3 und 4). Den Angaben zufolge ist die Beschäftigung mit dem Thema Inklusion in
Bayern somit stärker ausgeprägt als im Rheinland. Des Weiteren fallen die Werte bei Einrichtungen, die bereits $\mathrm{KmB}$ betreuen, in allen drei Kategorien höher aus als bei Einrichtungen, die noch keine KmB betreuen. Beide Einrichtungstypen geben in beiden Studien an, sich am häufigsten im Rahmen von Teambesprechungen mit Inklusion zu beschäftigen. Die größten Diskrepanzen zeigen sich hinsichtlich der konzeptionellen Überlegungen zum Thema Inklusion zwischen Einrichtungen mit $\mathrm{KmB}$ und denjenigen ohne KmB (vgl. Abb. 3 und 4).

\section{Diskussion zu Themenfeld 3}

In den teilnehmenden Kitas der RKS sind mehr als dreimal so viele Leitungen vom Gruppendienst freigestellt als in der IVO-Studie. Dies ist bemerkenswert, da Kita-Leitungen zunehmend eine zentrale Rolle für die Umsetzung von Inklusion und für die Qualitätsentwicklung in Kitas zukommt (Nentwig-Gesemann et al. 2016). Im Hinblick auf die zusätzlichen organisatorischen und fachlichen Aufgaben bei der Aufnahme von KmB ist die Frage einer zumin- 


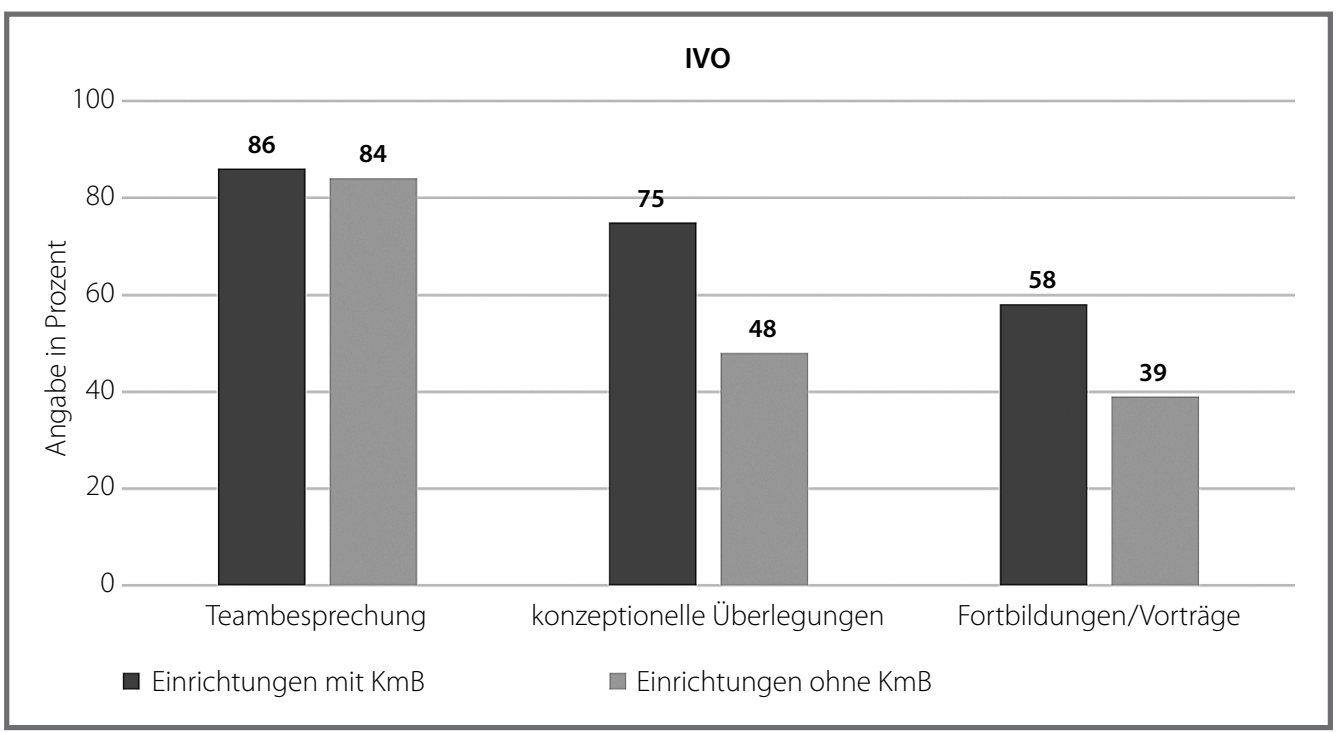

Abb. 3: Art der Beschäftigung mit dem Thema Inklusion in \% (IVO)

Anmerkung: Die Antworten von Einrichtungen mit KmB beziehen sich auf $\mathrm{N}=1.156$ Einrichtungen und von Einrichtungen ohne $\mathrm{KmB}$ auf $\mathrm{N}=756$ Einrichtungen.

dest zeitanteiligen Freistellung für Leitungsaufgaben zu überdenken. Insbesondere in Einrichtungen kleinerer Träger, bei denen die organisatorische Unterstützung durch über- geordnete Verwaltungsstrukturen meist geringer ausfällt, fallen für die Kita-Leitungen zusätzliche Aufgaben an, die für die Umsetzung von Inklusion wesentlich sind. Im Sinne einer

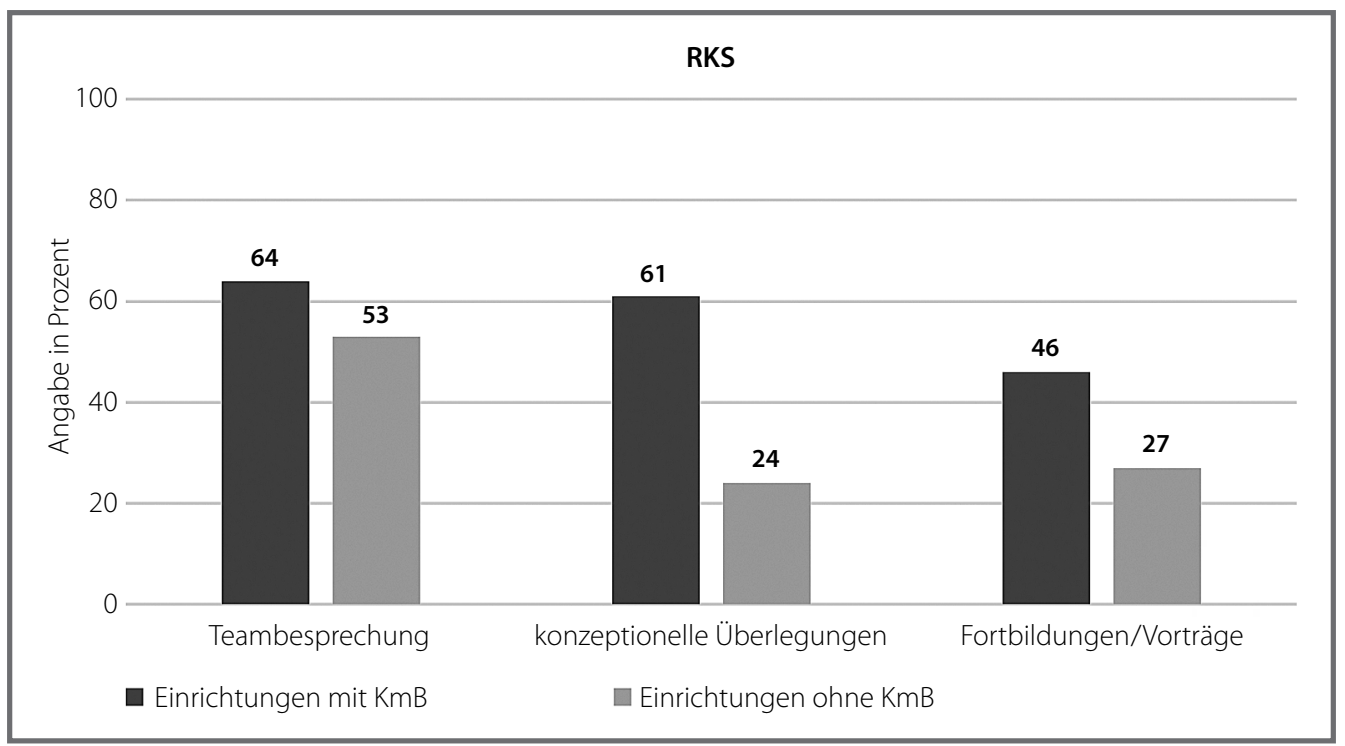

Abb. 4: Art der Beschäftigung mit dem Thema Inklusion in \% (RKS)

Anmerkung: Die Antworten von Einrichtungen mit KmB beziehen sich abhängig von den gewählten Antwortoptionen auf $\mathrm{N}_{\min }=455-$ $\mathrm{N}_{\max }=637$ Einrichtungen und von Einrichtungen ohne KmB auf $\mathrm{N}_{\min }=191-\mathrm{N}_{\max }=375$ Einrichtungen.<smiles></smiles> 
flächendeckenden Qualitätssicherung sind zudem entsprechende fachliche Aus-, Fort- und Weiterbildungen notwendig, um den komplexen Anforderungen an das wachsende Aufgabenspektrum der Kita-Leitungen gerecht werden zu können.

Zusammenfassend zeigen die Ergebnisse für Bayern und das Rheinland hinsichtlich der strukturellen Voraussetzungen in den inklusiven Kitas, dass Kita-Teams in Einrichtungen, die bereits $\mathrm{KmB}$ betreuen, in der Regel eine aufgeschlossenere Haltung zu dem Thema Inklusion aufweisen und sich intensiver mit der Umsetzung von Inklusion beschäftigen als KitaTeams in Einrichtungen ohne KmB. Dies wird insbesondere bei der Thematisierung von Inklusion in konzeptionellen Überlegungen bzw. bei dem Besuch von einschlägigen Fortbildungen deutlich. Offenbar steigt die Wahrscheinlichkeit, sich mit dem Thema Inklusion zu beschäftigen, deutlich mit der Aufnahme eines $\mathrm{KmB}$. Unstrittig scheint zudem der Mangel an spezifisch geschultem Personal zu dem Thema Inklusion sowohl in bayerischen als auch in rheinländischen Kitas zu sein. Die notwendige Ausrichtung auf inklusive Bildung innerhalb der Ausbildung zur Erzieherin bzw. zum Erzieher ist ein wichtiger Baustein. So erfordert die heterogener werdende Kinderschaft zusätzliches Fachwissen und therapeutische Unterstützung (Autorengruppe Bildungsberichterstattung 2018). Insofern scheint es sinnvoll, dass je nach Einrichtungsgröße mindestens eine Heilpädagogin bzw. ein Heilpädagoge, eine therapeutische Fachkraft oder eine Inklusionsfach- kraft auch Teil des Kita-Teams ist. Limitierend muss hier angemerkt werden, dass die Studien lediglich auf Befragungsdaten basieren und eine Qualitätsbeurteilung verschiedener Unterstützungsmodelle und deren Effektivität durch weitere Forschung geklärt werden muss.

\section{Kooperationsoptionen und Zufriedenheit mit den Kooperationen}

\section{Vernetzung mit Kooperations- partnerinnen und -partnern}

Die Kita-Leitungen wurden gebeten, aus einer Liste potenzieller Kooperationspartnerinnen und -partner diejenigen auszuwählen, zu denen sie Kontakt hatten. Mehrfachnennungen waren möglich. Bedingt durch länder- bzw. regionenspezifische Besonderheiten werden hier nicht sämtliche Antwortoptionen aus den Fragebögen berichtet. Tabelle 3 enthält stattdessen ausschließlich Angaben zu jenen Kooperationspartnerinnen und -partnern, zu denen in beiden Studien Daten vorliegen. Zu den weiteren Kooperationskontakten (z. B. verschiedene regional verfügbare Fachdienste) finden sich Angaben in den Projektberichten (Wölfl et al. 2017, Kißgen et al. 2019).

Zu den am häufigsten genannten Kooperationsoptionen zählen in beiden Studien Grundschulen, Fachberatungen sowie Fachtherapeutinnen und -therapeuten. In Bayern zeigen sich insgesamt deutlich weniger Kooperationen mit allen genannten Kooperationsoptionen.

Tab. 3: Kooperationspartnerinnen und -partner der Kindertageseinrichtungen

\begin{tabular}{|l|c|c|}
\cline { 2 - 3 } \multicolumn{1}{c|}{} & IVO & RKS \\
\hline Grundschulen & $85 \%$ & $98 \%$ \\
Fachberatung & $70 \%$ & $97 \%$ \\
Fachtherapie (z. B. Logopädie, Ergotherapie, Physiotherapie) & $70 \%$ & $91 \%$ \\
Familien- und Erziehungsberatungsstellen & $55 \%$ & $87 \%$ \\
Frühförderung & $54 \%$ & $88 \%$ \\
Medizinische und/oder psychologische Beratung & $28 \%$ & $78 \%$ \\
\hline
\end{tabular}




\section{Zufriedenheit mit den Kooperationen}

Hinsichtlich der Zufriedenheit mit den Kooperationen wurden in den Studien unterschiedliche Frageformate eingesetzt, sodass ein direkter Vergleich nicht möglich ist.

Mithilfe von Filterführung wurden die KitaLeitungen in Bayern gebeten, die Zusammenarbeit mit den Kooperationspartnerinnen und -partnern ihrer Kita in Hinblick auf Zufriedenheit zu bewerten. Die Fragen wurden nur den KitaLeitungen gestellt, die das entsprechende Angebot auch nutzen. Es handelte sich dabei um ein dichotomes Antwortformat (ja/nein). Die Zufriedenheit mit der fachlichen Unterstützung durch niedergelassene Fachtherapeutinnen und -therapeuten (84\%), Interdisziplinäre Frühförderstellen (84\%), Familien- und Erziehungsberatungsstellen (90\%) und Fachberatungen (91\%) fällt sehr hoch aus, während die Zufriedenheit mit Kooperationen aus dem medizinischen Bereich geringer ausfällt: Hier geben nur 69,3\% (Medizinische Beratung) bzw. 68,6\% (Sozialpädiatrische Zentren) an, mit der Kooperation zufrieden zu sein. Auch die Psychologische Beratung (71,9\%) erreicht etwas seltener positive Zustimmungswerte.

Die rheinländischen Kita-Leitungen konnten mit einer Skala von 1 („äußerst zufrieden“) bis 4 („äußerst unzufrieden") ihre Zufriedenheit mit den einzelnen Kooperationspartnerinnen und -partnern einschätzen. Wenn keine Kooperation bestand, konnte dies ebenfalls angekreuzt werden. Die höchsten Zufriedenheitswerte erreichten die Fachberatungen $(M=1.65, S D=0.67)$ sowie die Familien- und Erziehungsberatungsstellen ( $M=1.74, S D=0.62)$. Niedergelassene Fachtherapeutinnen und -therapeuten $(M=1.83$, $S D=0.61)$ und Frühförderstellen $(M=1.85, S D=$ $0.63)$ erzielten ebenfalls hohe Zufriedenheitswerte. Auch im Rheinland waren die Kita-Leitungen vergleichsweise weniger zufrieden mit Kooperationen, die mit Medizinischer Beratung $(M=2.4, S D=0.78)$ sowie mit Psychologischer oder Psychotherapeutischer Beratung bzw. Begleitung $(M=2.28, S D=0.73)$ einhergingen.

\section{Diskussion zu Themenfeld 4}

Durch länderspezifische und regional bedingte Unterschiede muss die Ergebnisdiskussion mit angemessener Zurückhaltung erfolgen. Festhalten lässt sich aber, dass die Häufigkeiten von Kooperationen im Rheinland insgesamt deutlich höher ausfallen als in Bayern.

Hinsichtlich der Fachberatung weist Hruska (2018) auf die bundesweit bestehende Heterogenität des Tätigkeitsfeldes hin, welche sich u. a. auf ihre Verortung und ihr Aufgabenspektrum erstreckt. Die hier referierten Ergebnisse untermauern zum einen die Relevanz der Fachberatung, da diese sowohl in der IVO-Studie als auch in der RKS als zweithäufigste Kooperationsoption genannt wird. Zudem wird die Zufriedenheit mit der fachlichen Unterstützung durch Fachberaterinnen und -berater in beiden Studien hoch eingeschätzt. Daraus lässt sich ableiten, dass Fachberatung trotz der systematischen Unklarheiten, die mit dieser Tätigkeitsbezeichnung einhergehen, eine wertvolle Bereicherung für viele Kitas darstellt.

Betrachtet man die Ergebnisse zu dem Bereich Frühförderung, fällt auch hier auf, dass diese Kooperationsoption im Rheinland deutlich häufiger in Anspruch genommen wird als in Bayern. Dies verwundert, da Frühfördereinrichtungen durch das Fachwissen und die konkreten therapeutischen Angebote ihres multiprofessionellen Teams wesentlich zur Bereicherung des kindbezogenen fachlichen Austauschs in den Kitas beitragen können. Gründe hierfür könnten in den unterschiedlichen länderspezifischen Frühfördersystemen oder der Angebotsdichte in Bayern und im Rheinland liegen. Während für die 8.748 Kitas in Bayern zum Zeitpunkt der Datenerhebung durch die IVO-Studie 198 Frühfördereinrichtungen inklusive der Außenstellen zur Verfügung standen, waren es im Rheinland während der Datenerhebung durch die RKS 162 Frühfördereinrichtungen für 5.220 Kitas. Es lässt sich somit vermuten, dass 
die geringere Inanspruchnahme von Frühförderstellen durch die bayerischen inklusiven Kitas im Wesentlichen durch das geringere Angebot zu erklären ist.

Die Kooperationsoptionen mit dem medizinischen und psychologischen Bereich werden in beiden Studien seltener in Anspruch genommen als andere Optionen und zugleich fällt die Zufriedenheit mit der fachlichen Unterstützung deutlich geringer im Vergleich mit anderen Optionen aus. Hürden für die Kooperation könnten darin bestehen, dass sich die Kommunikation zwischen pädagogisch ausgebildetem Fachpersonal und medizinischen oder psychologischen Fachkräften noch schwierig gestaltet. Des Weiteren könnten die Abrechnungssysteme für medizinische, psychologische und auch psychotherapeutische Leistungen die Kooperation mit einer Kita finanziell eher unattraktiv erscheinen lassen. Aufgrund des in diesen Disziplinen vorhandenen Fachwissens sollte jedoch zum Wohl der in den Kitas betreuten KmB dringend darüber nachgedacht werden, wie diese defizitäre Inanspruchnahme quantitativ und qualitativ verbessert werden kann.

\section{Bedeutung für die Praxis}

Insgesamt zeigen sowohl die IVO-Studie als auch die RKS deutlich, dass die Bereitschaft für inklusive Bildung im Elementarbereich vorhanden ist. Gleichzeitig lassen sich aber immer noch Hürden identifizieren. Zu diesen zählen beispielsweise das Hinterfragen und der Abbau wahrgenommener Barrieren. Anzugehen ist weiterhin die fachliche Stärkung der KitaTeams zum Thema Inklusion. In diesem Kontext kommt auch den externen Unterstützungssystemen eine besondere Bedeutung zu. Eine Kita darf nicht isoliert betrachtet werden. Innerhalb ihres Netzwerkes sollte ihr die Begleitung und Hilfestellung durch Fachberatungen und weitere Kooperationspartnerinnen und -partner zur Verfügung stehen.

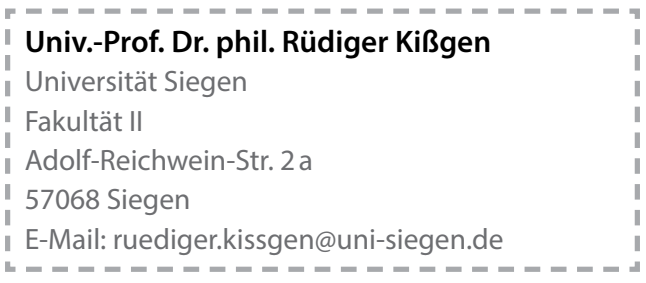

\section{Anmerkungen}

1 In NRW erfüllen zwei Landschaftsverbände (LVR für das Rheinland, LWL für die Region Westfalen-Lippe) überregionale kommunale Aufgaben. Sie sind Einrichtungen der kommunalen Selbstverwaltung und übernehmen u. a. als überörtlicherTräger der Jugendhilfe die Aufgaben des Landesjugendamtes (Landschaftsverbandordnung für das Land NRW - LVerbO).

2 KiBiz.web ist eine webbasierte Anwendung, in der u. a. Beantragung, Bewilligung und Abrechnung der Zuschüsse nach dem Kinderbildungsgesetz erfasst werden. Ebenfalls sind dort Informationen des Meldebogens nach §47 SGBVIII hinterlegt. (Meiner-Teubner und Sommer 2016)

3 Der Begriff "inklusiv“ bezieht sich hier auf inklusive und/oder integrative Kindertageseinrichtungen.

4 Cut-off-Frage:Eswurden nur Fragebögen einbezogen, die mindestens bis zu dieser Frage ausgefüllt wurden.

5 Landgemeinde < 5.000 Einwohner; Kleine Kleinstadt 5.001 - 10.000 Einwohner; Große Kleinstadt 10.001 20.000 Einwohner; Mittelstadt 20.001 - 100.000 Einwohner; Großstadt > 100.000.

6 Statistik der Kinder und tätigen Personen in Tageseinrichtungen. Anzahl der Einrichtungen insgesamt sowie Einrichtungen mit integrativer Betreuung und Einrichtungen für behinderte Kinder 2017 und 2018. Eigene Berechnungen.

7 Dazu zählen z. B. Heilpädagoginnen und Heilpädagogen, Heilerziehungspflegerinnen und -pfleger, Mitarbeiterinnen und Mitarbeiter mit einschlägiger Weiterbildung im Bereich Inklusion, Therapeutinnen und Therapeuten.

8 Dazu zählen z.B. Pädagoginnen und Pädagogen mit folgenden Schwerpunkten: Kindheitspädagogik, Heilpädagogik, Sozialpädagogik.

9 Ergotherapie, Logopädie, Physiotherapie oder Vergleichbares.

\section{Literatur}

Austermühle, J., Limburg, D., Wöhrle, J., Kißgen, R. (2019): Die Rheinland-Kita-Studie: Umsetzung von Inklusion in NRW - Teil 3. KiTa aktuell 28, 186-188 


\section{Autorengruppe Bildungsberichterstattung} (2018): Bildung in Deutschland 2018. Ein indikatorengestützter Bericht mit einer Analyse zu Wirkungen und Erträgen von Bildung. Bielefeld, https://doi.org/ 10.3278/6001820fw

Autorengruppe Bildungsberichterstattung (2014): Bildung in Deutschland 2014. Ein indikatorengestützter Bericht mit einer Analyse zur Bildung von Menschen mit Behinderungen. Bertelsmann, Bielefeld

Behr, I. (2009): Aspekte inklusiver Qualität in Kindertageseinrichtungen aus Sicht 4- bis 6-jähriger Kinder mit und ohne besondere Bedürfnisse. Eine Pilotstudie. Köster, Berlin

Brunner, J. (2018): Professionalität in der Frühpädagogik. Perspektiven pädagogischer Fachkräfte im Kontext einer inklusiven Bildung. Springer VS, Wiesbaden, https://doi.org/10.1007/978-3-658-20397-9_6

Heimlich, U. (2013): Kinder mit Behinderung Anforderungen an eine inklusive Frühpädagogik. Expertise der Weiterbildungsinitiative Frühpädagogische Fachkräfte (WiFF). München, https://doi.org/10. 1026/2191-9186/a000013

Hruska, C. (2018): Die Rolle der Fachberatung im System der frühkindlichen Bildung. In: https://www. kita-fachtexte.de/fileadmin/Redaktion/Publikationen/ KiTaFT_Hruska_2018-RollederFachberatung.pdf, 15.5.2020

ISB - Staatsinstitut für Schulqualität und Bildungsforschung (Hrsg.) (2015): Bildungsbericht Bayern. München, ISB

IT NRW Ref.512.Soziales.KJH (2020): Statistik der Kinder und tätigen Personen in Tageseinrichtungen. Anzahl der Einrichtungen insgesamt sowie Einrichtungen mit integrativer Betreuung und Einrichtungen für behinderte Kinder 2017 und 2018

Kißgen, R. (2019 a): Die Rheinland-Kita-Studie: Umsetzung von Inklusion in NRW - Teil 1. KiTa aktuell 28, $136-137$

Kißgen, R. (2019 b): Die Rheinland-Kita-Studie: Umsetzung von Inklusion in NRW - Teil 2. KiTa aktuell 28, $166-168$

Kißgen, R., Austermühle, J., Franke, S., Limburg, D., Wöhrle, J. (2019): Rheinland-Kita-Studie: Inklusion von Kindern mit Behinderung. Abschlussbericht. Universität Siegen. In: https://www.uni-siegen.de/fb2/ rheinlandkitastudie/abschlussbericht_rheinlandkita studie_final_190518.pdf, 7.4.2020

Kißgen, R., Austermühle, J., Wöhrle, J., Wiemert, H., Limburg, D. (im Druck): Betreuung von Kindern mit Behinderung in den Tageseinrichtungen für Kinder des Rheinlandes - Ergebnisse der Rheinland-KitaStudie. VHN
Mayr, T., Held, L. (2010): RisKid Zwischenbericht. In: http://www.ifp.bayern.de/projekte/monitoring/ riskid.php, 7.4.2020

Meiner-Teubner, C., Sommer, A. (2016): Das Wissen über Kitas - Können landesinterne Verwaltungsdaten die KJH-Statistik ergänzen? KomDat 1/2016, 10-13

Nentwig-Gesemann, I., Nicolai, K., Köhler, L. (2016): Kita-Leitung als Schlüsselposition. Erfahrungen und Orientierungen von Leitungskräften in Kindertageseinrichtungen. In: https://www.bertels mann-stiftung.de/fileadmin/files/BSt/Publikationen/ GrauePublikationen/Studie_WB_Kita-Leitung_als_ Schluesselposition_2016.pdf, 8.7.2020

Schelle, R. (2017): Die Kita-Leitung als Schlüssel für Inklusion? Eine Analyse beruflicher Kompetenzen und Arbeitsbedingungen. Zeitschrift Für Inklusion, (4) In: https://www.inklusion-online.net/index.php/ inklusion-online/article/view/389, 18.5.2020

Statistisches Landesamt Bayern (2015): Statistischer Bericht zu Kindertageseinrichtungen und Kindertagespflege. Bayerisches Landesamt für Statistik und Datenverarbeitung, München. In: https://www. statistik.bayern.de/statistik/bildung_soziales/kinder_ jugend_hilfe/index.html\#link_7, 8.7.2020

Wertfein, M., Wirts, C. (2016): Inklusion als gemeinsame Aufgabe von Kindertageseinrichtung und Frühförderung. In: Gebhard, B., Seidel, A., Sohns, A., MöllerDreischer, S. (Hrsg.): Frühförderung mittendrin - in Familie und Gesellschaft. Stuttgart, Kohlhammer, $162-170$

Wirts, C., Wertfein, M., Wengert, C., Frank, C. (2015): Lust und Mut zur Inklusion in Kindertageseinrichtungen. Handreichung zur Öffnung von Kindertageseinrichtungen für Kinder mit Behinderung. In: https:// www.ifp.bayern.de/imperia/md/content/stmas/ifp/ lust-und-mut-inklusion-kita_barrierefrei.pdf, 10.6.2020

Wirts, C., Wertfein, M., Wölfl, J. (2018): IVO - Eine Studie zur Umsetzung von Inklusion als gemeinsame Aufgabe von Kindertageseinrichtungen und Frühförderung in Bayern. Vernetzungsbericht. IFP-Projektbericht 33/2018. Staatsinstitut für Frühpädagogik, München. In: https://www.ifp.bayern.de/imperia/md/ content/stmas/ifp/projektbericht_33_ivo_vernetzung_ barrierefrei.pdf, 7.4.2020

Wölfl, J., Wertfein, M., Wirts, C. (2017): IVO - Eine Studie zur Umsetzung von Inklusion als gemeinsame Aufgabe von Kindertageseinrichtungen und Frühförderung in Bayern. Kita-Ergebnisbericht. IFP-Projektbericht 30/2017. Staatsinstitut für Frühpädagogik, München. In: https://www.ifp.bayern.de/imperia/md/ content/stmas/ifp/projektbericht_ivo_nr_30.pdf, 7.4.2020 